\title{
Phase unwrapping for large depth-of-field 3D laser holographic interferometry measurement of laterally discontinuous surfaces
}

\author{
Zhenhua Huang ${ }^{1}$, Albert J Shih ${ }^{2}$ and Jun $\mathrm{Ni}^{2}$ \\ ${ }^{1}$ Coherix Inc., Ann Arbor, MI 48108, USA \\ ${ }^{2}$ Department of Mechanical Engineering, University of Michigan, Ann Arbor, MI 48109, \\ USA \\ E-mail: zhenhuah@coherix.com
}

Received 17 July 2006, in final form 2 September 2006

Published 19 October 2006

Online at stacks.iop.org/MST/17/3110

\begin{abstract}
A phase unwrapping method is developed to mathematically increase the depth-of-field for the 3D optical measurement of objects with laterally discontinuous surfaces, which contain disconnected high aspect ratio regions. This method is applied for laser holographic interferometry precision measurements. The phase wrap identification at boundary pixels, masking and recovery, dynamic segmentation and phase adjustment are developed to overcome the divergence problem in phase unwrapping of laterally discontinuous surfaces. An automotive automatic transmission valve body is applied as an example to demonstrate the developed method. Experimental results demonstrate that the proposed methods can efficiently unwrap the phase to increase the depth-of-field for laterally discontinuous surfaces. Effects of segment size and width of overlapped regions on the computational efficiency are investigated.
\end{abstract}

Keywords: phase unwrapping, laser holographic interferometry, laterally discontinuous surfaces

(Some figures in this article are in colour only in the electronic version)

\section{Introduction}

Phase unwrapping is a mathematical procedure to eliminate the ambiguity in the phase map for imaging applications, including the synthetic aperture radar interferometry, laser holographic interferometry, magnetic resonance imaging and others [1]. In this research, phase unwrapping is studied to increase the depth-of-field for the 3D laser holographic interferometry measurement of laterally discontinuous surfaces. An example of an object with laterally discontinuous surfaces is the automatic transmission valve body, as shown in figure $1(a)$. Figure 1(b) shows the top view of the measurement surface of a valve body, which contains grooves for pressurized fluid to control the position of valves for gear shifting. The flatness and precision height measurement of the surface is important for the sealing of an automatic transmission.

Phases calculated by laser holographic interferometry range from $-\pi$ to $\pi$, the principal value range of reverse trigonometric functions. The limited range of phase creates the phase wrap and ambiguity in measurement. Figure 2(a) shows an example of a parabolic-shaped surface with phases ranging from 0 to $18 \mathrm{rad}$. The measurement of this surface with phase wraps is shown in figure $2(b)$. Sudden changes of phase are observed at pixels with the phase value near $-\pi$ or $\pi$. Phase unwrapping restores the true phase map (figure 2(a)) from the measured phase map (figure $2(b)$ ). If the spatial sampling rate in the phase map is at least twice the highest frequency of the change of phase, so-called Shannon sampling theorem [2], a phase wrap is assumed when the phase difference between 


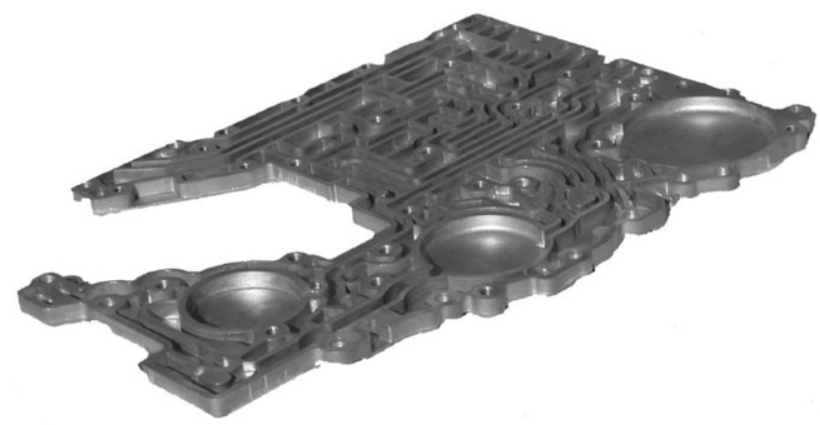

(a)

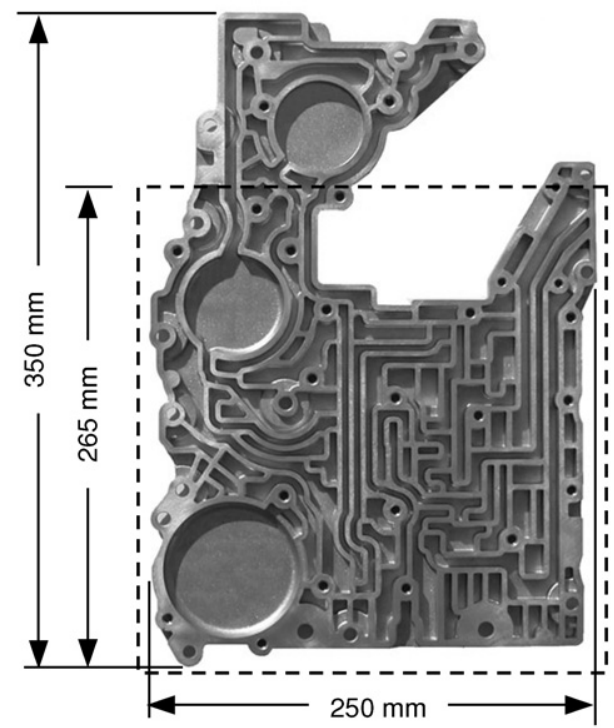

(b)

Figure 1. The automatic transmission valve body: $(a)$ isometric view and $(b)$ top view.

two adjacent pixels exceeds a threshold value of $\pi$. The wrapped phase is compensated by the integral multiples of $2 \pi$ to be unwrapped.

Technical advancements in phase unwrapping have been aimed at achieving high noise robustness and low computational cost. Both temporal- and spatial-based approaches for phase unwrapping have been developed. Temporal-based phase unwrapping, developed by Huntley and Saldner [2, 3], unwraps the change of phase over time for each pixel independently. The error is restricted to individual pixels and does not propagate between pixels. Special optical configurations [4] are required to relate the unwrapped phase to the height of the surface. This makes the temporal-based approach unsuitable for the laser holographic interferometry measurement, which is the targeted application in this research.

Spatial-based phase unwrapping processes the phase in the 2D spatial domain using either path-dependent or pathindependent methods. The path-dependent method unwraps the phase along a specially designed path by converting the $2 \mathrm{D}$ array into a folded 1D data set. This method needs complex path design strategy in the presence of noise [5] and is not feasible for the measurement of the laterally discontinuous surface.

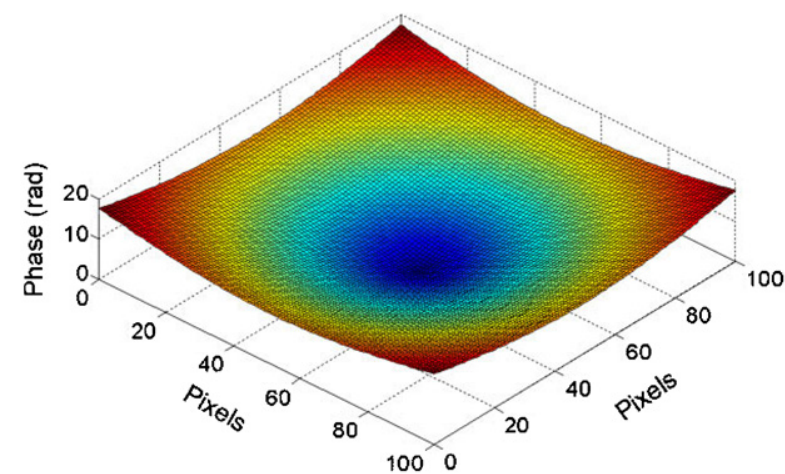

(a)

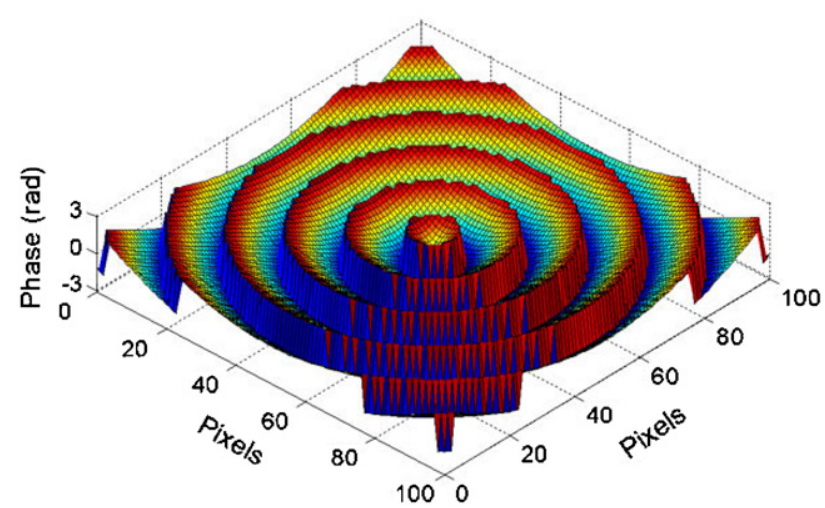

(b)

Figure 2. Artificially created phase map: (a) 3D true phase map without phase wrap and $(b)$ 3D wrapped phase map.

The path-independent methods, including model- $[6,7]$, Bayesian- [8], least-squares- [9], and integration-based [10-18], do not require a specially designed path for spatialbased phase unwrapping. However, none of these pathindependent methods can be directly applied to unwrap the phase of laterally discontinuous surfaces because of the narrow, curved regions and the discontinuity among regions. The region-referenced method [18], which is an integrationbased path-independent method, uses the phase data in regions surrounding a pixel to detect the phase wrap. This method has good noise robustness, can automatically adjust the direction to adapt for narrow and curved regions, and was selected to be further developed in this study.

Laser holographic interferometry is reviewed in section 2. The region-referenced phase unwrapping, segmentation and patching, and problems for narrow segmented regions and boundary pixels are explained in section 3. In section 4, the solutions of phase wrap identification on boundary pixels, masking and recovery, dynamic segmentation, and phase adjustment are developed. An example is presented in section 5 to validate the proposed method and study the computational efficiency and convergence.

\section{Laser holographic interferometry}

The configuration of laser holographic interferometry for precision height measurement is shown in figure 3 . The 


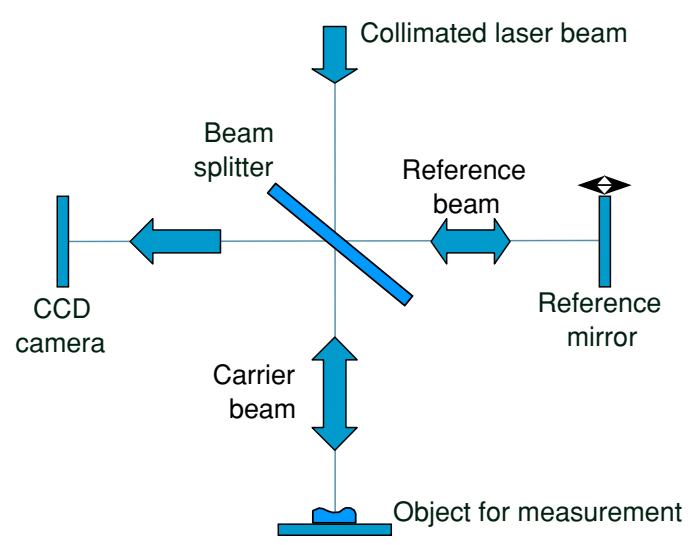

Figure 3. Diagram of the laser holographic interferometer.

collimated laser beam is split by the beam splitter into two coherent laser beams, denoted as the reference beam and carrier beam. The reference beam reflects from the reference mirror back to the beam splitter. The carrier beam reflects from the object surface and has the phase modulated by the height of the object. The reflected carrier beam and the reference beam interfere with each other and generate the interference pattern, which is recorded as a hologram in the charged-coupled device (CCD) camera.

The phase-shifting and multi-wavelength tuning methods are applied to calculate the height of the object surface [19]. The phase, which is linearly proportional to the surface height, is extracted from the recorded hologram in the CCD camera. The height measurement range with the phaseshifting technology is half of the wavelength. According to the Shannon sampling theorem [2], the height difference between two adjacent pixels in the CCD camera must be smaller than $1 / 4$ of the laser wavelength for phase unwrapping. This is too small for most practical applications. The multiwavelength technology [19] has been applied to increase the height measurement range. The height of each point on the surface is calculated by tuning the laser wavelength and Fourier transforming the change of phases which are obtained using the phase-shifting technology at each wavelength. The range of height measurable by laser holographic interferometry using the multi-wavelength tuning depends on the incremental wavelength tuning step. The smaller the tuning step, the larger the height measurement range can be. However, the tradeoff for the increase in the height measurement range using a smaller wavelength tuning step is the decrease of resolution and accuracy.

Phase unwrapping can overcome this problem. A large wavelength tuning step is applied in the multi-wavelength method to obtain a wrapped phase map with high resolution and accuracy. The phase unwrapping method then unwraps the phase map to obtain a larger height measurement range while maintaining the high resolution and accuracy.

\section{Region-referenced phase unwrapping}

The region-referenced method is applied as the base for the developed phase unwrapping method. The region-referenced

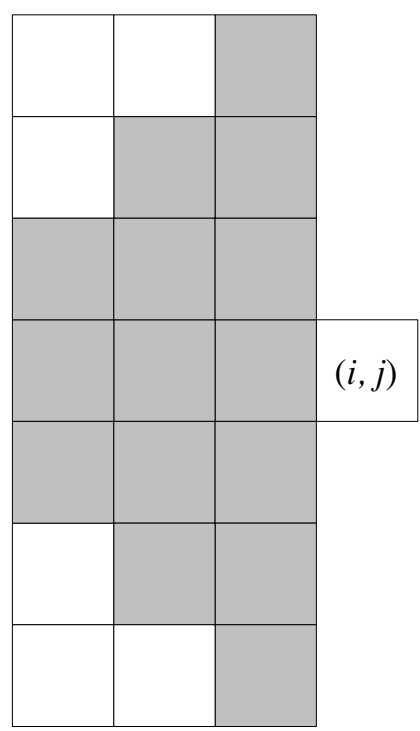

(b)

Figure 4. Detection of phase wrap at pixel $(i, j)$ : $(a)$ surrounding four pixels and $(b)$ reference region surrounding the pixel.

method uses an iterative algorithm to unwrap the phase. This method searches the pixels with phase wraps and compensates the phase by adding or subtracting $2 \pi$ depending on the sign of the phase wrap. The surface is assumed continuous in the height direction with no phase jump over $\pi$ between two adjacent pixels. The searching and compensation processes are repeated until no phase wrap is detected.

\subsection{Principle}

A simple method to determine the phase wrap is to use the four adjacent pixels of a pixel, marked as $(i, j)$ in figure $4(a)$, to determine the phase wrap [5, 20]. If at least one of the four adjacent pixels has a phase difference larger than $\pi$, this pixel is recognized to have phase wrap and needs to be compensated by adding or subtracting $2 \pi$ for phase unwrapping. The problem of this phase unwrapping method is the sensitivity to noise, which can cause the divergence of the iteration. A more complicated region-referenced criterion was developed by Huang and He [18] to overcome this problem. Each adjacent pixel in figure $4(a)$ is replaced by a region to determine the phase wrap. For example, the adjacent pixel $(i-1, j)$ in figure $4(a)$ is replaced by 15 pixels, as shown by the shaded area in figure $4(b)$, to determine the phase wrap. If over half of the pixels in the shaded region have a phase difference larger than $\pi$ with the pixel $(i, j)$, a phase wrap is identified at pixel $(i, j)$. The criterion of over half of pixels, which is eight in the case in figure 4(b), is empirically optimal [18]. Rotating the shaded region around the pixel $(i, j)$ by $90^{\circ}, 180^{\circ}$, and $270^{\circ}$, the other three reference regions corresponding to the other three adjacent pixels in figure 4(a) can be obtained. The phase wrap at pixel $(i, j)$ is identified if any of the four reference regions have phase wrap. Other reference regions of different shapes are also developed [18]. This method can significantly improve the noise robustness. However, pixels on the boundary, so-called boundary pixels, have limited numbers 


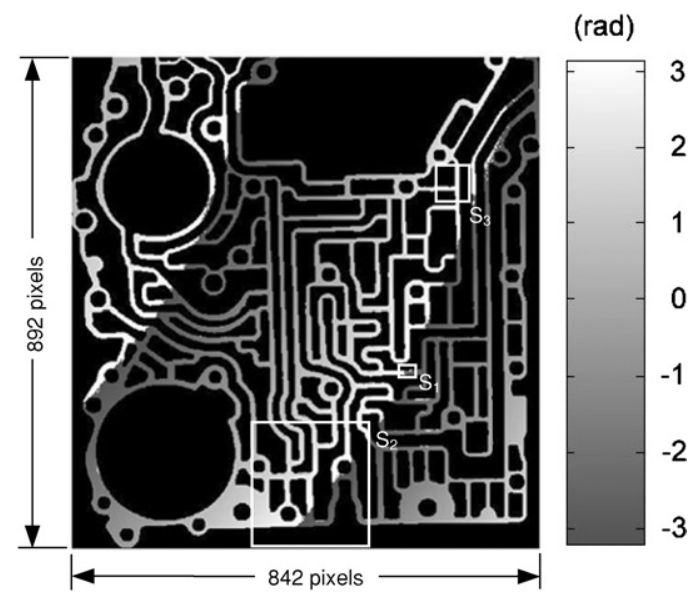

Figure 5. Measured phase map with phase wrap for the automatic transmission valve body.

of pixels in the reference region to detect the phase wrap. This makes boundary pixels more sensitive to noise.

A laterally discontinuous surface, like the automatic transmission valve body in figure 1 , has many boundary pixels due to the discontinuity between regions, and can introduce the divergence problem in phase unwrapping. Figure 5 shows the measured phase map of the automatic transmission valve body. The solid black background is the region not for measurement. All the phase values are within the range from $-\pi$ to $\pi$, the principal value range of reverse trigonometric functions. Phase wraps are observed in the phase map. The close-up view of the region $\mathrm{S}_{1}$ is shown in figure $6(a)$. The boundary pixels, marked by arrows, are a source of instability and make it easy for the region-referenced phase unwrapping to diverge. The idea proposed in this research is to first conduct the phase unwrapping without considering these boundary pixels, i.e., boundary pixels with phase wrap are masked. After phase unwrapping, these masked pixels are recovered by median filtering. This concept will be elaborated in section 4.1.

\subsection{Segmentation and patching}

Segmentation, which divides the phase map into many small overlapped segments, has been developed to improve the computational efficiency for phase unwrapping [16, 18]. Each segment is first unwrapped independently. Then, the data of two adjacent segments are connected using the overlapped region between these two segments. Static segmentation is defined as the segmentation method with fixed segment size and width of overlapped regions during unwrapping.

Patching is the process of combining the data of individual segments after phase unwrapping into an integral phase map. Two adjacent segments with an overlapped region are compared with each other and the phases of one segment are shifted by a multiple of $2 \pi$ to make the overlapped regions of the two segments match. After shifting, these two adjacent segments are concatenated. This procedure is applied to all the adjacent segments until an integral phase map including all the individual segments is reached. The path which the patching

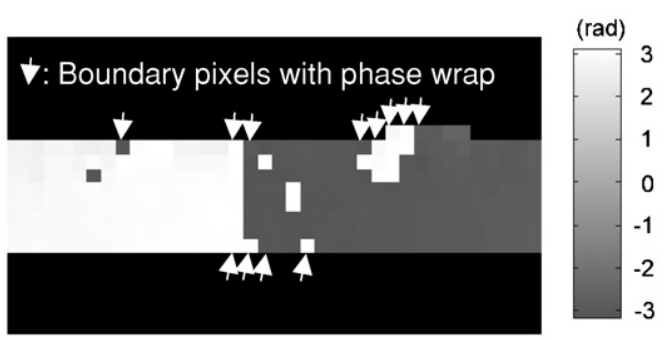

(a)

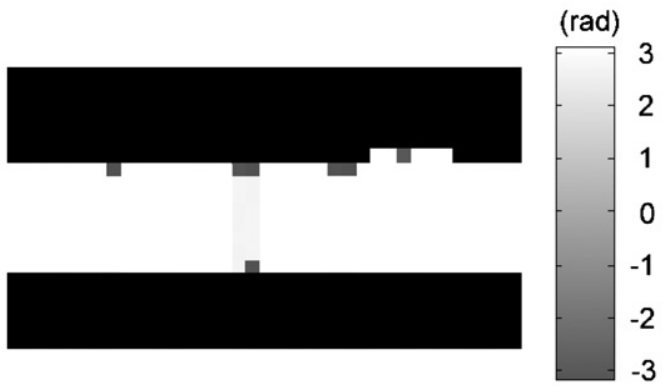

(b)

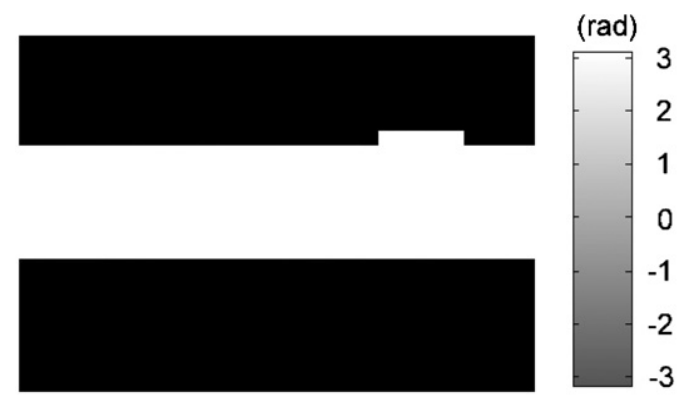

(c)

Figure 6. Close-up view of the region $S_{1}$ in figure 5 as an example of the boundary pixels with decreased noise robustness in the detection of phase wrap: ( $a$ ) before phase unwrapping, $(b)$ after phase unwrapping and before recovery and $(c)$ after phase unwrapping and recovery.

process follows is determined iteratively by systematically selecting one of the four adjacent segments as the next segment to be patched.

An example of static segmentation with patching is shown in figure 7(a), which illustrates the close-up view of region $\mathrm{S}_{2}$ of the valve body in figure 5 . The size of each segment is $(W+$ $\left.W_{o}\right) \times\left(W+W_{o}\right)$ with the width of the overlapped region equal to $W_{o}$. The unwrapped phase in $\mathrm{C}_{1} \mathrm{D}_{1} \mathrm{E}_{1} \mathrm{~F}_{1}$ and $\mathrm{C}_{2} \mathrm{D}_{2} \mathrm{E}_{2} \mathrm{~F}_{2}$ will be patched to make the phase in overlapped region $\mathrm{C}_{2} \mathrm{D}_{1} \mathrm{E}_{1} \mathrm{~F}_{2}$ match both $\mathrm{C}_{1} \mathrm{D}_{1} \mathrm{E}_{1} \mathrm{~F}_{1}$ and $\mathrm{C}_{2} \mathrm{D}_{2} \mathrm{E}_{2} \mathrm{~F}_{2}$.

The problem of the static segmentation for laterally discontinuous surfaces is the thin boundary regions. An example of the thin boundary region is shown in figure 7(b), which is the close-up view of the region $\mathrm{S}_{4}$ in figure $7(a)$. A boundary region with only 1 to 2 pixels in width after static segmentation and patching is observed on the top of the segment. The phase wrap which exists in this boundary region cannot be detected or removed using the phase unwrapping method. The dynamic segmentation, to be discussed in section 4.3 , is developed to overcome this problem. 


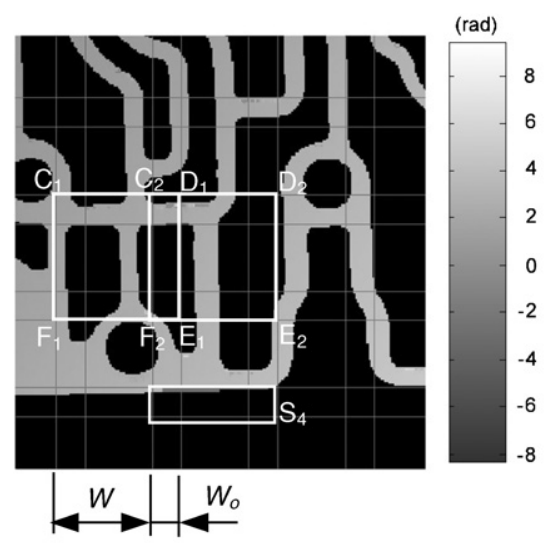

(a)

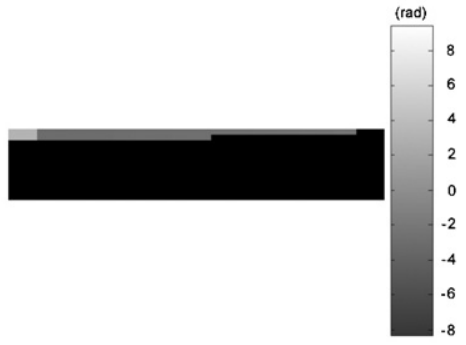

(b)

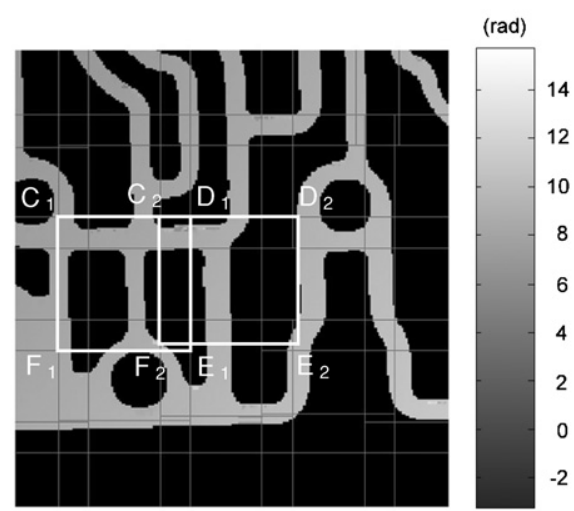

(c)

Figure 7. Segmentation: (a) static segmentation of the region $\mathrm{S}_{2}$ in figure $5,(b)$ close-up view of the region $\mathrm{S}_{4}$ in figure $7(a)$, and (c) dynamic segmentation of the region $\mathrm{S}_{2}$ in figure 5 .

\section{Masking, dynamic segmentation and adjustment}

The masking and recovery, dynamic segmentation and phase adjustment are developed to improve the robustness and the computational efficiency for phase unwrapping of laterally discontinuous surfaces. To avoid the divergence problem, boundary pixels with phase wrap are masked during the phase unwrapping. Dynamic segmentation adaptively determines the segment size to reduce the number of thin boundary regions in segmentation. The phase adjustment recovers the phase value of masked pixels to complete the phase unwrapping.

\subsection{Phase wrap identification on boundary pixels}

A criterion is developed to determine if a boundary pixel has phase wrap. Each boundary pixel has four reference regions. In each reference region, some pixels are not on the object surface and do not have valid phase information. Those pixels with valid phase information are denoted as valid pixels. If more than half of the valid pixels in any of the four reference regions of a boundary pixel have phase difference larger than $\pi$, this boundary pixel is designated to have phase wrap and will be masked in the phase unwrapping.

\subsection{Masking and recovery}

Masking is applied to the boundary pixels with phase wrap to avoid divergence. The masked boundary pixels with phase wrap are not processed for the phase unwrapping. However, if the masked boundary pixel is still a valid pixel and its phase information is used in reference regions of other pixels for phase unwrapping analysis.

After phase unwrapping, to recover the value at a masked boundary pixel, median filtering [21] is applied. Median filtering first sorts the phase values of pixels in a matrix (usually $3 \times 3,5 \times 5,7 \times 7$, or $9 \times 9$ in dimension) centred at the pixel to be filtered in a sequence and then chooses the median as the new phase value. If any pixel in the matrix has been masked, the values of these pixels are not included in the sorting sequence. Median filtering has the advantage of suppressing the spike noise.

An example of the masking and recovery process is illustrated in figure 6. Figure 6(a) shows a close-up view of the region $S_{1}$ in figure 5 . Boundary pixels with phase wrap are marked by arrows. These pixels are masked for the phase unwrapping. Figure $6(b)$ shows the result after phase unwrapping and before recovery. Seven masked boundary pixels still have phase wrap before the recovery. The recovery process assigns phase values to the masked pixels using a $7 \times 7$ median filter. The result after applying the median filtering is shown in figure $6(c)$. The phase unwrapping is completed without a divergence problem. Phase values of masked boundary pixels have been recovered. 


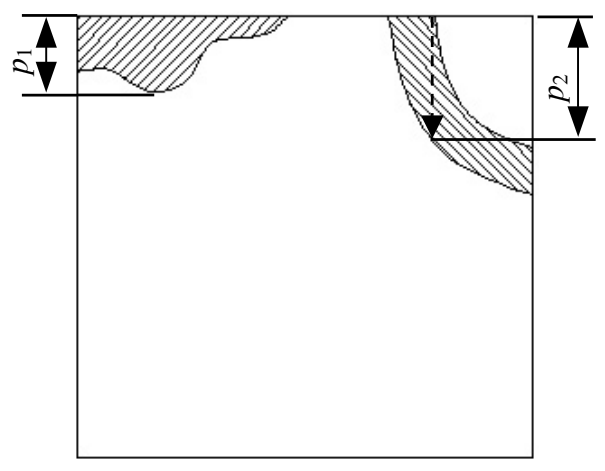

(a)

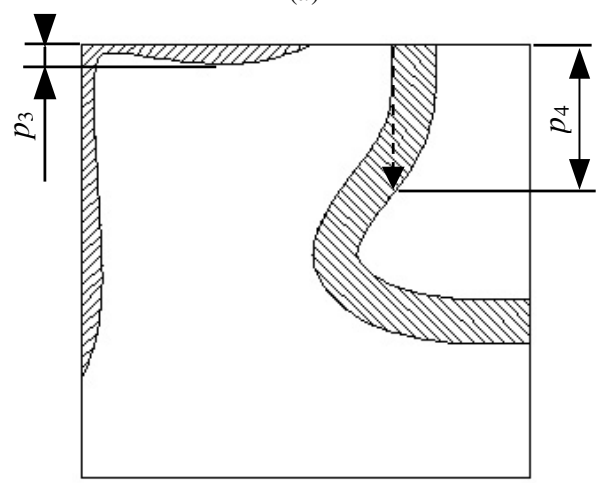

(b)

Figure 8. Definition of the boundary width $p$ : $(a)$ two boundary regions and $(b)$ the condition with a thin boundary region close to the left side of the segment.

\subsection{Dynamic segmentation}

The static segmentation, as discussed in section 3.2, has fixed segment size and fixed width of overlapped regions. Dynamic segmentation is developed in this study to overcome the divergence problem caused by thin boundary regions of laterally discontinuous surfaces.

To identify the thin boundary region, the width of boundary regions needs to be quantified. A parameter, called the boundary width $p$, of each boundary region is defined as the maximum number of pixels inside the boundary region in the direction perpendicular to the side of the segment. An example is shown in figure $8(a)$. Two unmasked boundary regions, marked by hatched lines, have $p$ equal to $p_{1}$ and $p_{2}$, respectively. The smaller of the two, $p_{1}$, is denoted as the minimum side width $p_{\min }$, which is defined as the minimum of the boundary widths of all the boundary regions on a side of the segment.

An exception in the determination of $p$ occurs when a thin boundary region exists along the other two adjacent sides. As shown in figure $8(b)$, if pixels in the direction perpendicular to the side of the segment are very close, less than a predetermined threshold $T$, to the other side of the segment, these pixels are not considered in the calculation of $p$. In figure $8(b)$, the smaller of $p_{3}$ and $p_{4}$ is used to determine the $p_{\min }$ to represent the minimum boundary width of the top side of the rectangular segment.

Dynamic segmentation starts with the static segmentation. If $p_{\text {min }}$ of a segment is smaller than $T$ after the static segmentation, dynamic segmentation is triggered. The side of the segment after the static segmentation will be moved towards the outside of the segment by $m_{1}=\left(T-p_{\text {min }}\right)$ pixels to increase the width of the thin boundary region.

The counting of $p_{\min }$ is applied again to identify if any thin boundary region still exists on the new segment. If it does, the side of the segment is moved in the opposite direction by $m_{2}=$ $m_{1}+T / 2$ pixels. This dynamic segmentation is repeated on all four sides of the rectangular segment and has demonstrated the ability to eliminate most, but not all, of the thin boundary regions in practical applications.

Figure 9 shows an example of the dynamic segmentation of region $\mathrm{S}_{3}$ in figure 5. The segment size and the width of overlapped regions are $W=50$ and $W_{o}=20$ pixels in the static segmentation. The original segment after the static segmentation is shown in figure $9(a)$. The segment including the overlapped region is $70 \times 70$ pixels. The top side of the segment has two regions. The $p$ in the middle region is 70 and in the right region is 2 , which is smaller than the predetermined threshold value $T(=6)$ and is identified as a thin boundary region. $p_{\min }$ is equal to 2 . Using the dynamic segmentation, the top side of the segment is then moved upward by $m_{1}=4$ $(=6-2)$ pixels. The newly obtained segment, as shown in figure $9(b)$, has a new thin boundary region with the boundary width $p(=4)$ in the upper left corner. The top side of the segment is then moved in the opposite direction by $m_{2}=7$ $(=4+6 / 2)$ pixels. The newly obtained segment with no thin boundary region on the top side is shown in figure $9(c)$. The same procedure is repeated on the other three sides. The final segment after the dynamic segmentation is $67 \times 74$ pixels, as shown in figure $9(d)$.

The phase unwrapping results with dynamic segmentation of the region $S_{2}$ in figure 5 are shown in figure $7(c)$. For the segment $C_{1} D_{1} E_{1} F_{1}$ with no thin boundary region, the segment after dynamic segmentation, $\mathrm{C}_{1}^{\prime} \mathrm{D}_{1}^{\prime} \mathrm{E}_{1}^{\prime} \mathrm{F}_{1}^{\prime}$, does not change. The segment $\mathrm{C}_{2} \mathrm{D}_{2} \mathrm{E}_{2} \mathrm{~F}_{2}$ has thin boundary regions. The dynamic segmentation changes the segment to $\mathrm{C}_{2}^{\prime} \mathrm{D}_{2}^{\prime} \mathrm{E}_{2}^{\prime} \mathrm{F}_{2}^{\prime}$ to eliminate the thin boundary regions. The phase wrap in region $\mathrm{S}_{4}$ of figure $7(a)$ is eliminated after dynamic segmentation (figure $7(c)$ ).

\subsection{Phase adjustment for thin boundary regions with phase wrap}

After dynamic segmentation, some thin boundary regions may still exist. The phase wrap in these thin boundary regions cannot be solved using the region-referenced method described in section 3. The phase adjustment method is developed to identify the phase wrap and adjust the phase values in the integral phase map by adding or subtracting a multiple of $2 \pi$ to complete the phase unwrapping process.

Figure 10(a) illustrates two examples of regions $\mathrm{S}_{5}$ and $\mathrm{S}_{6}$ in the integral phase map of the automatic transmission valve body (figure 2). The close-up view of the region $\mathrm{S}_{5}$ is shown in figure $10(b)$. The thin boundary region in $S_{5}$ is located on the top side. During dynamic segmentation, the boundary line cannot extend beyond the integral phase map and the thin boundary region remains. The close-up view of $\mathrm{S}_{6}$ is shown in figure $10(c)$. The thin boundary region with phase wrap still exists after moving the boundary line twice in dynamic segmentation. 


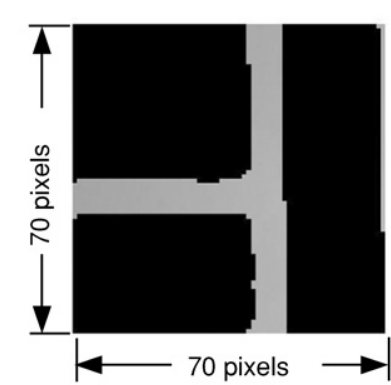

(a)

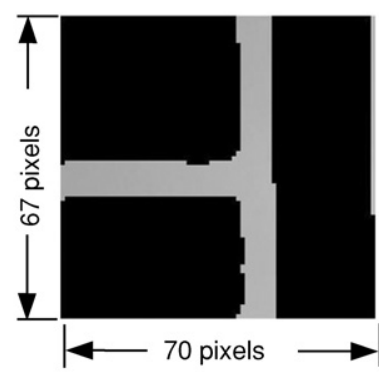

(c)

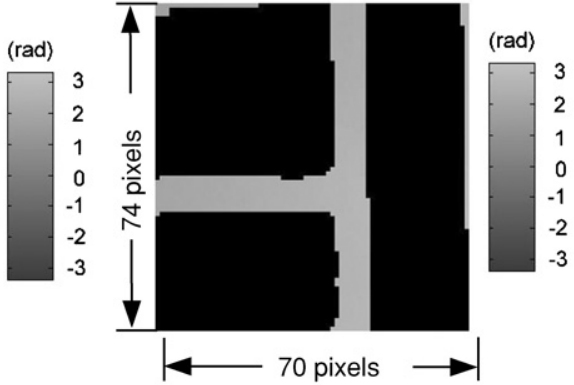

(b)
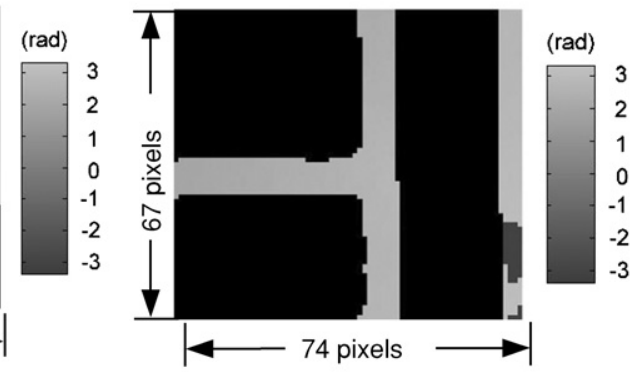

(d)

Figure 9. Example of the dynamic segmentation of region $\mathrm{S}_{3}$ in figure $5\left(W=50, W_{o}=20\right.$, and $T=6$ pixels).

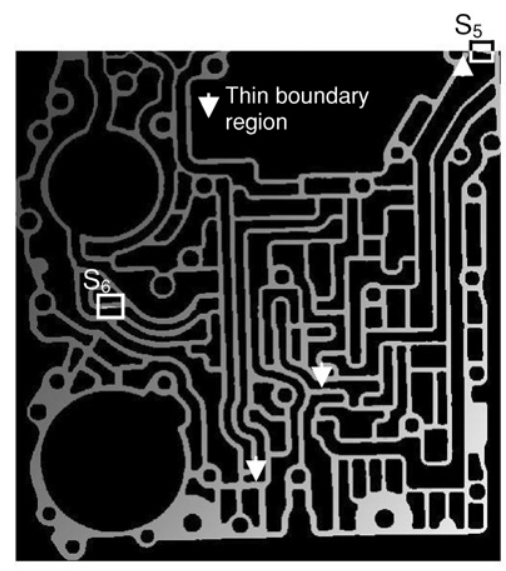

(a)

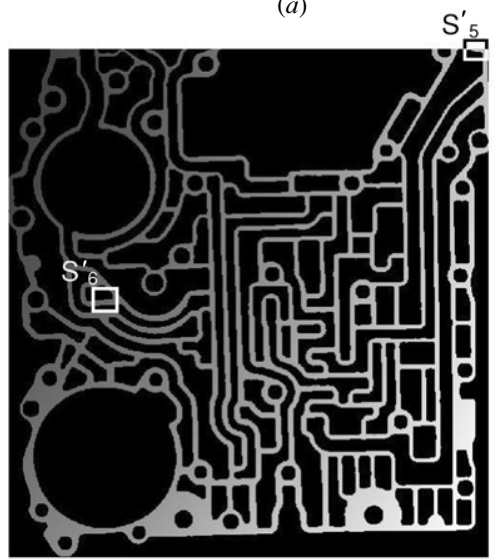

$(d)$
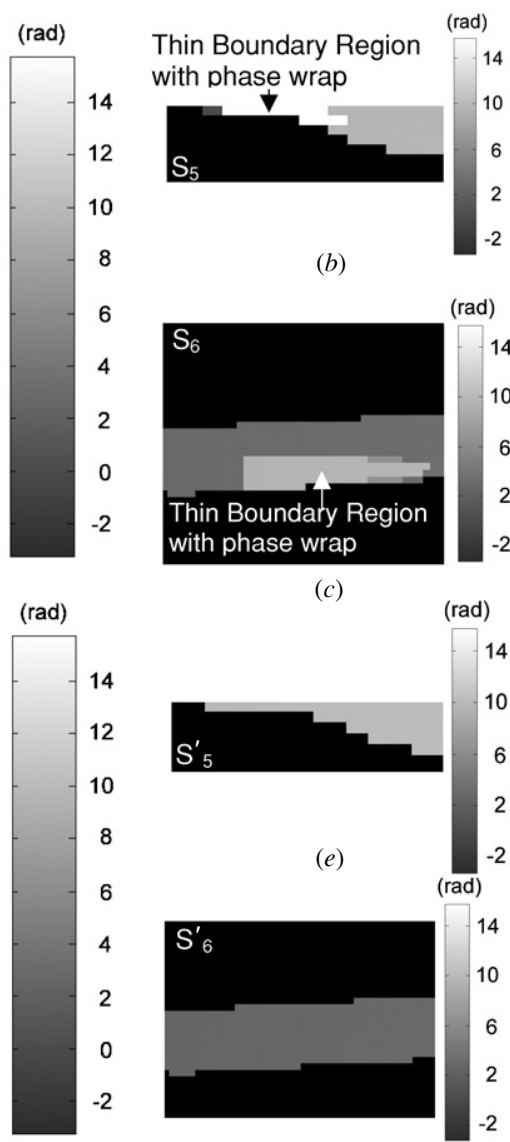

$(f)$

Figure 10. Phase unwrapping of the automatic transmission valve body: $(a)$ unwrapped phase map without adjustment, $(b)$ close-up view of the region $\mathrm{S}_{5}$ in $(a),(c)$ close-up view of the region $\mathrm{S}_{6}$ in $(a),(d)$ unwrapped phase map after adjustment, $(e)$ close-up view of the region $\mathrm{S}_{5}^{\prime}$ in $(d)$ after adjustment, and $(f)$ close-up view of the region $\mathrm{S}_{6}^{\prime}$ in $(d)$ after adjustment. 
The phase values of pixels in thin boundary regions can be estimated or extrapolated using a polynomial function fitted by the data of the integral phase map. The estimated phase values are compared to the existing phases of pixels in thin boundary regions to determine the adjustment in the multiple of $2 \pi$. The polynomial function can be of the first, second or higher order. The first-order polynomial function representation of the integral phase map is

$$
\varphi_{1}(i, j)=a_{0,1}+a_{1,1} i+a_{2,1} j,
$$

where $i$ and $j$ are the row and column coordinates, respectively, of the integral phase map, $\varphi_{1}(i, j)$ is the estimated phase value of the pixel $(i, j)$ in the integral phase map, and $a_{0,1}, a_{1,1}$ and $a_{2,1}$ are coefficients of the first-order polynomial function.

Coefficients $a_{0,1}, a_{1,1}$ and $a_{2,1}$ are determined using the least square estimation [22]:

$\left\{\begin{array}{l}a_{0,1} \\ a_{1,1} \\ a_{2,1}\end{array}\right\}=\left[\begin{array}{ccc}\sum 1 & \sum i & \sum j \\ \sum i & \sum i^{2} & \sum i j \\ \sum j & \sum i j & \sum j^{2}\end{array}\right]^{-1}\left\{\begin{array}{c}\sum \varphi_{1} \\ \sum i \varphi_{1} \\ \sum j \varphi_{1}\end{array}\right\}$.

The second-order polynomial function of $\varphi(i, j)$ is

$\varphi_{2}(i, j)=a_{0,2}+a_{1,2} i+a_{2,2} j+a_{3,2} i^{2}+a_{4,2} i j+a_{5,2} j^{2}$,

where $a_{0,2}, a_{1,2}, a_{2,2}, a_{3,2}, a_{4,2}$ and $a_{5,2}$ are estimated by [22]

$$
\begin{aligned}
\left\{\begin{array}{l}
a_{0,2} \\
a_{1,2} \\
a_{2,2} \\
a_{3,2} \\
a_{4,2} \\
a_{5,2}
\end{array}\right\}= & {\left[\begin{array}{cccccc}
\sum 1 & \sum i & \sum j & \sum i^{2} & \sum i j & \sum j^{2} \\
\sum i & \sum i^{2} & \sum i j & \sum i^{3} & \sum i^{2} j & \sum i j^{2} \\
\sum j & \sum i j & \sum j^{2} & \sum i^{2} j & \sum i j^{2} & \sum j^{3} \\
\sum i^{2} & \sum i^{3} & \sum i^{2} j & \sum i^{4} & \sum i^{3} j & \sum i^{2} j^{2} \\
\sum i j & \sum i^{2} j & \sum i j^{2} & \sum i^{3} j & \sum i^{2} j^{2} & \sum i j^{3} \\
\sum j^{2} & \sum i j^{2} & \sum j^{3} & \sum i^{2} j^{2} & \sum i j^{3} & \sum j^{4}
\end{array}\right] } \\
& \times\left\{\begin{array}{c}
\sum \varphi_{2} \\
\sum i \varphi_{2} \\
\sum j \varphi_{2} \\
\sum i^{2} \varphi_{2} \\
\sum i j \varphi_{2} \\
\sum j^{2} \varphi_{2}
\end{array}\right\} .
\end{aligned}
$$

To determine which order polynomial is adequate for phase adjustment, the root mean square, $e_{\mathrm{rms}}$, of the estimation error is applied.

$$
e_{\mathrm{rms}}=\sqrt{\frac{\sum_{i, j}\left(\varphi(i, j)-\varphi_{k}(i, j)\right)^{2}}{N}},
$$

where $k$ is the order of the polynomial function and $N$ is the total number of data points in the integral phase map.

If $e_{\mathrm{rms}}$ is small than a predefined threshold value, for example, $0.2 \mathrm{rad}$, which was experimentally verified to be sufficient in this study, the polynomial function is considered adequate for the phase adjustment. Otherwise, higher order polynomial functions will be fitted using the least square method [22] until the $e_{\mathrm{rms}}$ is smaller than the threshold value.

\section{Example}

The measurement of the surface on an automatic transmission valve body, as shown in figure 1 , is used as an example of a laterally discontinuous surface to demonstrate the developed phase unwrapping method. The measurement area, as shown in figure $1(b)$, was $265 \times 250 \mathrm{~mm}$. The measurement result was represented in a matrix with the dimensions of $892 \times 842$ pixels, as shown in figure 5 . The height measurement range was $0.3 \mathrm{~mm}$. The lower right corner of the valve body was raised by a wedge to increase the height range of the valve body surface beyond $0.3 \mathrm{~mm}$ to generate the phase wrap.

\subsection{Phase unwrapping results}

To unwrap the measured phase map shown in figure 5, the region-referenced phase unwrapping method described in section 3 is applied. The 15 pixels reference region, as shown in figure $4(b)$, is utilized to identify the phase wrap. For each pixel, if more than eight pixels in any of the four reference regions have a phase difference larger than $2 \pi$, that pixel is marked to have phase wrap. Masking and the $7 \times 7$ median filter for recovery, as discussed in section 4.2, are applied to those boundary pixels with phase wrap to avoid the divergence problem on boundary pixels.

The dynamic segmentation with $W=50$ pixels, $W_{o}$ $=15$ pixels, and $T=6$ pixels, is applied to generate $18 \times 17(=306)$ segments. This segmentation generates 71 thin boundary regions. The dynamic segmentation is ${ }_{-1}$ applied to move all four sides (top, bottom, right and left) of the segments to reduce the number of thin boundary regions. In this example, 53 segments have at least one of the four sides moved once and three segments have at least one of the four sides moved twice. After the dynamic segmentation, only five thin boundary regions exist. These remaining thin boundary regions are marked by three arrows and two boxes $\left(\mathrm{S}_{5}\right.$ and $\left.\mathrm{S}_{6}\right)$ in figure $10(a)$. Figures $10(b)$ and $(c)$ illustrate close-up views of the thin boundary regions in $\mathrm{S}_{5}$ and $\mathrm{S}_{6}$.

The dynamic segmentation was necessary to achieve a converged solution to phase unwrapping in this example. If only the static segmentation was used, the integral phase map was incorrect and the phase adjustment could not correct the errors in the integral phase map.

To recover the phase values in the five thin boundary regions shown in figure $10(a)$, the first- and second-order polynomial functions were applied to fit the integral phase map. Results of the fitted polynomial functions are $\varphi_{1}(i, j)=$ $-1.521+0.006 i+0.013 j$ with $a_{0,1}=-1.521, a_{1,1}=0.006$ and $a_{2,1}=0.013$, and $\varphi_{2}(i, j)=-0.497+0.003 i+0.010 j+$ $4 \times 10^{-6} i^{2}+1 \times 10^{-7} i j+4 \times 10^{-6} j^{2}$ with $a_{0,2}=-0.497, a_{1,2}=$ $0.003, a_{2,2}=0.010, a_{3,2}=4 \times 10^{-6}, a_{4,2}=1 \times 10^{-7}$ and $a_{5,2}$ $=4 \times 10^{-6}$.

The root mean square $e_{\mathrm{rms}}$ is calculated to determine which order of the polynomial function is adequate for the phase adjustment. The total number of data points $N$ is 191131 . The $e_{\mathrm{rms}}$ for the first- and second-order polynomials are $0.33 \mathrm{rad}$ and $0.16 \mathrm{rad}$, respectively. Because the $e_{\mathrm{rms}}$ of the second-order polynomial is smaller than the predefined threshold value of $0.2 \mathrm{rad}$, this polynomial is adequate for the phase adjustment.

The difference is calculated between the existing and estimated values of $\varphi_{1}(i, j)$ in the thin boundary regions. The multiple of $2 \pi$ is added to or subtracted from those pixels in the thin boundary regions to make the difference smaller than $2 \pi$. For example, in $S_{5}$ and $S_{6}, 2 \pi$ was subtracted from both 
Table 1. Computational time for phase unwrapping of the automatic transmission valve body example $(892 \times 842$ pixels $)$.

\begin{tabular}{llll}
\hline $\begin{array}{l}\text { Segment size, } \\
W \text { (pixels) }\end{array}$ & $\begin{array}{l}\text { Width of } \\
\text { overlapped regions, } \\
W_{o} \text { (pixels) }\end{array}$ & $\begin{array}{l}\text { Number of } \\
\text { segments }\end{array}$ & $\begin{array}{l}\text { Computational } \\
\text { time (min) }\end{array}$ \\
\hline 150 & 25 & 36 & 38 \\
100 & 25 & 81 & 25 \\
50 & 25 & 306 & 18 \\
40 & 25 & 506 & 15 \\
30 & 25 & 870 & 15 \\
29 & 25 & 930 & 16 \\
$<29$ & 25 & & Divergence \\
40 & 20 & 506 & 12 \\
40 & 15 & 506 & 9 \\
40 & 10 & 506 & 6 \\
40 & $<10$ & 506 & Divergence \\
\hline
\end{tabular}

regions to bring the phase values from the level of $15 \mathrm{rad}$ to $9 \mathrm{rad}$ and from $9 \mathrm{rad}$ to $3 \mathrm{rad}$, respectively. Figure $10(d)$ shows the integral phase map after the phase adjustment. The close-up views of the two regions $S_{5}^{\prime}$ and $S_{6}^{\prime}$ in figure $10(d)$ are illustrated in figures $10(e)$ and $(f)$, respectively. The errors have been removed and the whole phase unwrapping process is complete.

\subsection{Effect of $W$ and $W_{o}$ on computational efficiency}

Two sets of numerical experiments to study the effect of $W$ and $W_{o}$ on computational efficiency are conducted and results are summarized in table $1 . W_{o}$ is fixed at 25 pixels in the first set of tests. The reduction of $W$ from 150 to 30 pixels improves the computational time from 38 to $15 \mathrm{~min}$ using a common personal computer with MATLAB software. A smaller size segment requires less computational time for phase unwrapping. However, reducing $W$ increases the number of segments and eventually raises the computational time. As shown in table 1 , when $W$ is reduced to 29 pixels, the computational time is increased to $16 \mathrm{~min}$. When $W$ is smaller than 29 pixels, the phase unwrapping method diverges.

The second set of numerical experiments is conducted by fixing $W$ and reducing $W_{o}$. With a fixed $W$ at 40 pixels, as shown in table 1 , the reduction of $W_{o}$ from 25 to 10 pixels further reduces the computational time to $6 \mathrm{~min}$ while the number of segments remains the same at 506. As $W_{o}$ reduces, the segment size after dynamic segmentation becomes smaller and makes the phase unwrapping of each segment faster. The total computational time is reduced. When $W_{o}$ is smaller than 10 pixels ( $W=40$ pixels), a divergence problem occurs.

\subsection{Convergence}

The convergence of the phase unwrapping method is also investigated. As shown in table 2, for $W$ at 50, 40 and 29, the minimum $W_{o}$ without a divergence problem are 10, 10 and 24 , respectively, for the automatic transmission valve body example. When $W$ reduces from 50 to 40 pixels, the minimum $W_{o}$ is the same (10 pixels) to make the phase unwrapping method converge. When $W$ reduces from 40 to 29 pixels, the minimum $W_{o}$ increases to 24 pixels. This is due to the increase in the number of boundary pixels in smaller segments
Table 2. Minimum $W_{o}$ for phase unwrapping without divergence in the automatic transmission valve body example.

\begin{tabular}{ll}
\hline$W$ (pixels) & $\begin{array}{l}\text { Minimum } W_{o} \text { (pixels) } \\
\text { without divergence }\end{array}$ \\
\hline 50 & 10 \\
40 & 10 \\
29 & 24 \\
\hline
\end{tabular}

making the region-referenced phase unwrapping more prone to divergence.

This example demonstrates the importance of selecting $W$ and $W_{o}$ to improve computational efficiency without compromising the stability in phase unwrapping. The selection of $W$ and $W_{o}$ will change for different applications. The basic principle learned in this example is to use an optimal $W$ which balances the number of segments and computational time in each segment. Small $W_{o}$ without triggering instability is desired.

\section{Concluding remarks}

A new phase unwrapping method was developed to mathematically increase the height measurement range of laser holographic interferometry while maintaining the same level of accuracy and resolution. This method had successfully solved the phase unwrapping problem of laterally discontinuous surfaces using the example of an automatic transmission valve body. Three new methods, including masking and recovery, dynamic segmentation and phase adjustment for pixels in thin boundary regions, have been demonstrated to be effective to avoid the divergence problem of boundary pixels. A guideline was developed to select the size of segment to improve the computational efficiency without incurring a convergence problem.

This phase unwrapping method, together with the hologram registration method developed by Huang et al [19] to expand the field of view, has successfully increased the measurement volume for laser holographic interferometry measurements.

\section{Acknowledgments}

This work was supported by the Engineering Research Center for Reconfigurable Manufacturing Systems of the National Science Foundation under Award Number EEC-9529125. The technical support of Dwight Carlson, Ron Swonger and Mike Mater of Coherix Inc. is greatly appreciated.

\section{References}

[1] Strand J, Taxt T and Jain A K 1999 Two-dimensional phase unwrapping using a block least-squares method IEEE Trans. Image Process. 8 375-86

[2] Huntley J M and Saldner H 1993 Temporal phase-unwrapping algorithm for automated interferogram analysis Appl. Opt. 32 3047-52

[3] Huntley J M and Saldner H O 1997 Shape measurement by temporal phase unwrapping: comparison of unwrapping algorithms Meas. Sci. Technol. 8 986-92 
[4] Saldner H O and Huntley J M 1997 Temporal phase unwrapping: application to surface profiling of discontinuous objects Appl. Opt. 36 2770-5

[5] Gasvik K J 2002 Optical Metrology (Hoboken, NJ: Wiley)

[6] Friedlander B and Francos J M 1996 Model based phase unwrapping of 2-D signals IEEE Trans. Signal Process. 44 2999-3007

[7] Liang Z P 1996 A model-based method for phase unwrapping IEEE Trans. Med. Imaging $15893-7$

[8] Leitao J and Figueiredo M A 1995 Interferometric image reconstruction as a nonlinear Bayesian estimation problem Proc. Int. Conf. on Image Processing pp 453-6

[9] Ghiglia D C and Romero L A 1994 Robust two-dimensional weighted and unweighted phase unwrapping that uses fast transforms and iterative methods J. Opt. Soc. Am. A 11 $107-17$

[10] Huntley J M and Coggrave C R 1998 Progress in phase unwrapping Proc. SPIE-The International Society for Optical Engineering vol 3407, pp 86-93

[11] Huntley J M 1989 Noise-immune phase unwrapping algorithm Appl. Opt. 28 3268-70

[12] Cusack R, Huntley J M and Goldrein H T 1995 Improved noise-immune phase-unwrapping algorithm Appl. Opt. 34 781-9

[13] Buckland J R, Huntley J M and Turner S R E 1995 Unwrapping noisy phase maps by use of a minimum-cost-matching algorithm Appl. Opt. 34 5100-8
[14] Quiroga J A, Gonzalez-Cano A and Bernabeu E 1995 Stable-marriages algorithm for preprocessing phase maps with discontinuity sources Appl. Opt. 34 5029-38

[15] Gutmann B and Weber H 2000 Phase unwrapping with the branch-cut method: role of phase-field direction Appl. Opt. 39 4802-16

[16] Chang H Y, Chen C W, Lee C K and Hu C P 1998 The tapestry cellular automata phase unwrapping algorithm for interferogram analysis Opt. Lasers Eng. 30 487-502

[17] Strand J and Taxt T 2002 Two-dimensional phase unwrapping using robust derivative estimation and adaptive integration IEEE Trans. Image Process. 10 1192-200

[18] Huang M J and He Z N 2002 Phase unwrapping through region-referenced algorithm and window-patching method Opt. Commun. 203 225-41

[19] Huang Z, Shih A J and Ni J 2006 Laser interferometry hologram registration for three-dimensional precision measurements J. Manuf. Sci. Eng. Trans. ASME (at press)

[20] Stephenson P, Burton D R and Lalor M J 1994 Data validation techniques in a tiled phase unwrapping algorithm Opt. Eng. Bellingham 33 3703-8

[21] Gonzalez R C and Woods R E 2002 Digital Image Processing (Upper Saddle River, NJ: Prentice-Hall)

[22] Wu C F J and Hamada M 2000 Experiments: Planning, Analysis, and Parameter Design Optimization (New York: Wiley) 